\title{
Initially Clamped Piezoelectric Inchworm Linear Motor based on Force Amplification Mechanisms Design for Miniaturized and Large Force Actuation Applications
}

\author{
Hussam Kloub \\ Mechanical and Me dical Enginee ring Department, Furtwangen University, Robert-Gerwig-Platz 1, 78120 \\ Furtwangen, Germany; hussam.kloub@hs-furtwangen.de; Tel.: +49-7723-920-2944
}

\begin{abstract}
In this paper, a novel monolithic structural design of PZT inchw orm motor utilizing three Force-Amplification-Mode mechanisms is presented as an approach to overcome design challenges of common PZT inchworm motors. A mechanical system model based on Simulink software was developed for a proposed inchworm motor design. The dynamic response of the motor was simulated at the moment of releasing the pre-stressed mechanism. The results showed a backlash response due to the mass acceleration of the mechanisms.
\end{abstract}

Keywords: Piezoelectric motor; Piezoelectric inchworm; Amplification Mechanism; Force Amplification Mechanism; Miniaturized Motor

\section{Introduction}

Piezoelectric inchworm linear motor are common devices in high precision positioning applications, such in optical equipment and precision manufacturing. Inchworm motors can be realized with one or multiple piezoelectric (PZT) stacks. The positioning of an object is performed either by a direct contact of PZT elements that are functioning in shear mode [1-2], or by indirect contact, through flexural mechanisms, where the PZT elements are functioning in normal mode [34]. The main advantage of using flexural mechanism is to enhance the reliability of PZT element against damaging. Fortunately, flexural mechanisms can be also designed to amplify the generated displacement or force by the PZT stack, for example by applying mechanical lever design concept. However, miniaturization, large-force actuation capability, large internal stresses, fragile flexural design based on lever concept and requirement for high precision manufacturing are main challenges for realizing PZT inchw orm motor based on amplified mechanisms design .

The new design consists of three main integrated mechanisms, such that each is driven by single PZT element. Two clamping mechanism, initially confined by a mechanical guidance, and become released when the corresponding PZT element is electrically polarized. The other mechanism is centered between the clamping units, and perform device stretching when the corres ponding PZT element is electrically polarized.

\section{Methods}

\subsection{Structural Design and Operation Concept}

The top view of the initially clamped inchw orm motor based on force amplification mode (FAM) mechanisms is shown in Figure 1. The piezoelectric inchworm motor consists of three main units, two for clamping function and one for extending function. The clamping units are confined by a 
mechanical guidance to provide initial clamping condition. The mechanism at the center of the motor is responsible for providing extending function.

Each mechanism consists of flexural structure and a piezoelectric stack placed at the center of the mechanism. When the piezoelectric stack is electrically polarized then a corresponding expansion deformation occurs in the axis of the piezoelectric stack force, while compression defeormation occurs in the normal angle to the axis of the piezoelectric stack force.

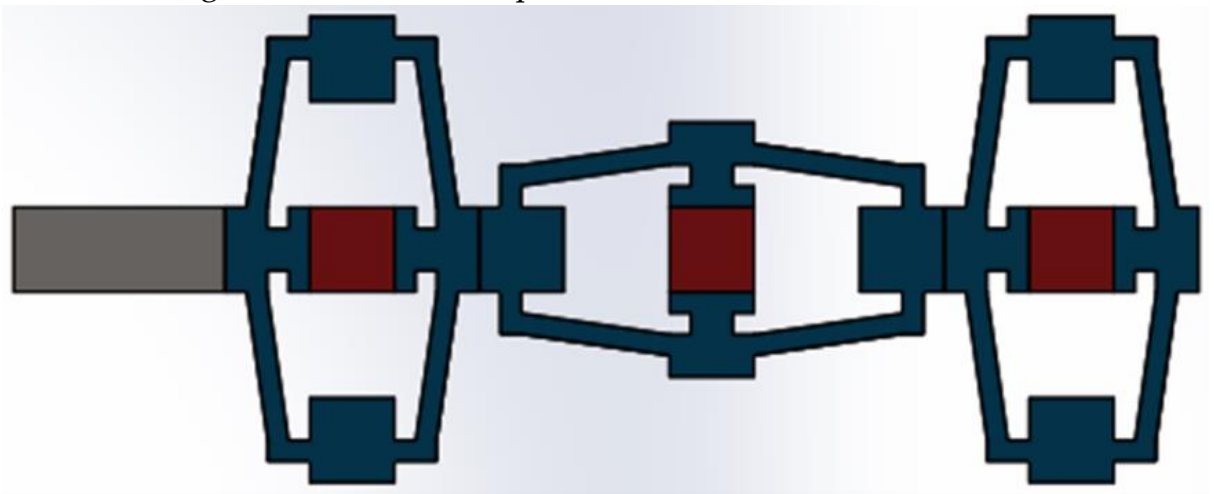

Figure 1. Top view of a piezoelectric inchworm motor based on force a mplification mode design.

A motor stroke is generated by the cooperative work of the three mechanisms. Initially, the motor is clamped by the mechanical guidance. When the piezoelectric stack of one clamping unit is electrically polarized, then the mechanical contanct of the mechanism is detached from the mechanical guidance. At this moment, the piezoelectric stack of the central mechanism (extending mechanism) is electrically polarized. This will pull the released clamping unit tow ard the central mechanism. After that, the clamping unit is returned to mechanical attachment with the mechanical guidance by electrically deactivating the corresponding piezoelectric stack. Then the other clamping unit is detached from the mechanical guidance, and the piezoelectric stack of the central mechanism is now electrically deactivated to allow the mechanism returning back to the initial mechanical condition, which also results in mechanical translation of the recently released clamping unit, before it is returned back to mechanical clamping status. This process is repeated for further stroke actuation, in order to achive a longer distance actuation.

\subsection{System Modelling and Boundary Conditions}

The presented inchworm motor can be modelled based on three coupled oscillating masses as shown in Figure 2. The model was developed to study the dynamic response of the motor at the extending status; i.e. the piezoelectric stacks of clamping unit 1 and the extending unit (central mechanism) are initially activated. When the piezoelectric stack of the extending unit is deactivated, the stored elastic energy will be released causing acceleration of the three mass blocks. The stored elastic energy is defined by the initial mechanical deflection $\left(\mathrm{S}_{\mathrm{A}}\right)$ and stiffness constant $(\mathrm{K})$ of each mechanical spring.

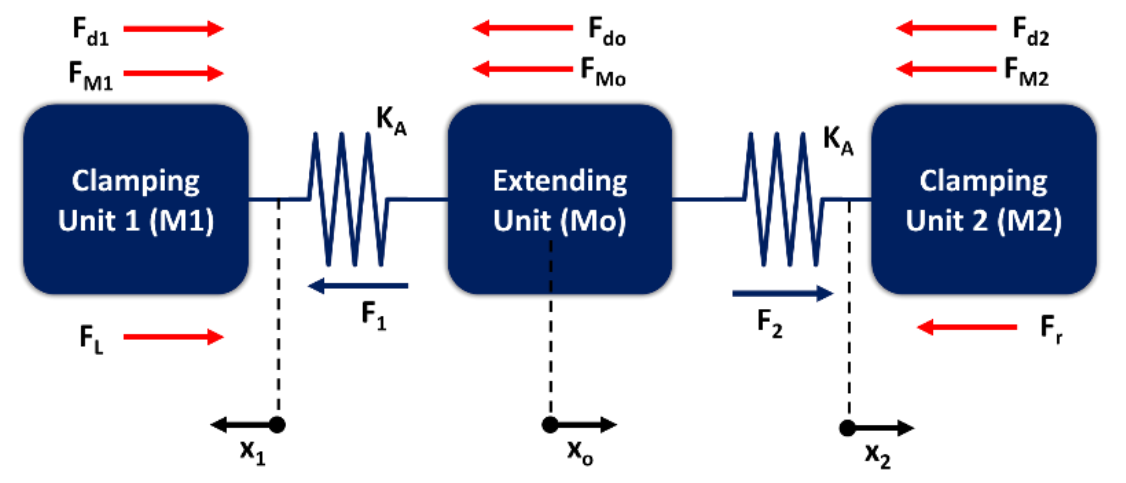

Figure 2. Mass-Spring model of force amplification mode pie zoelectric inchworm motor. 
The coupled oscillating mass model can be mathematically represented by:

$$
\begin{gathered}
x_{o}=0.5\left(x_{2}-x_{1}\right), \\
a_{1}=\frac{1}{m_{1}}\left(K_{A}\left(S_{A}-\left(x_{1}+x_{o}\right)\right)+m_{o} a_{o}+b\left(v_{o}-v_{1}\right)-F_{L}\right), \\
a_{o}=\frac{1}{m_{o}}\left(m_{1} a_{1}-m_{2} a_{2}+b\left(v_{1}-v_{2}-v_{o}\right)+F_{L}-F_{r}\right), \\
a_{2}=\frac{1}{m_{2}}\left(K_{A}\left(S_{A}-\left(x_{2}-x_{o}\right)\right)-b v_{2}-F_{r}\right),
\end{gathered}
$$

The parameters values of stiffness constant $\left(\mathrm{K}_{\mathrm{A}}\right)$ and inititial displacements $\left(\mathrm{S}_{\mathrm{A}}\right)$ of elastic elements depend on the structural design of the mechanical advantage mechanism and the electromechanical characetristics of the applied PZT element. The specific structural design of the mechanical advantage mechanism is not the focus of this work, therefore arbitrary values will be selected that apply a force amplification mode. In this work, a PZT element with $1.25 \mu \mathrm{m}$ stroke at $125 \mathrm{~N}$ generated force w as arbitrary assumed. Accor dingly, the $S_{A}$ and $K_{A}$ values are $0.125 \mu \mathrm{m}$ and 10 $\mathrm{GN} / \mathrm{m}$ for a FAM design with a mechanical advantage factor of 10 . A mass magnitude of $0.2 \mathrm{~g}$ was selected for the three mass blocks. The damping coefficient was set to $100 \mathrm{~N} . \mathrm{s} / \mathrm{m}$. A friction force of $200 \mathrm{~N}$ w as assumed at the clamping unit 2. The equations of the model were solved based on Simulink model in Figure 3.

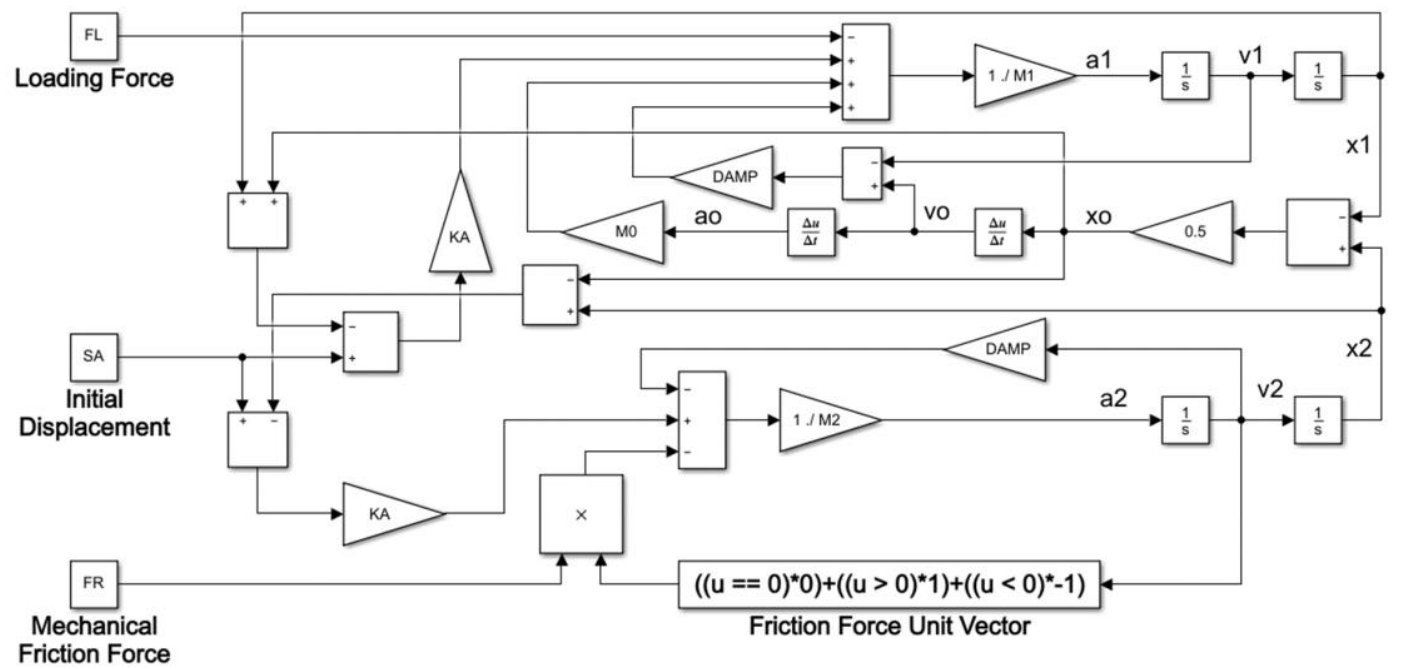

Figure 3. Simulink block diagram of the mechanical model of the piezoelectric inchworm motor.

\section{Results and Discussion}

The dynamic mechanical response of inchw orm motor is shown in Figure 4. The final stroke is a result of the vector summation of deflections of all mass components. The releasing of elastic energy affects both the clamping and the loading mass blocks, such that deflection in both directions is observed. This response reduces the final stroke of the inchw orm motor. 


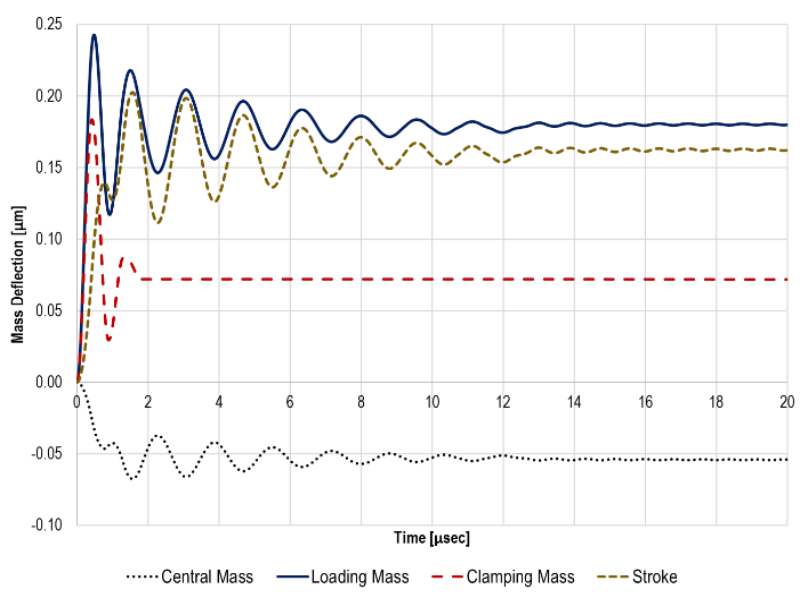

Figure 4. Displacementsimulation results of FAM piezoelectric inchworm motor under 200 Nfirction force and ze ro loa ding force.

\section{Conclusions}

A monolithic design of a piezoelectric inchworm motor initially clamped by a mechanical guidance was proposed in this paper. The main advantage of the new design is that all mechansims for clamping and extending function are based on force amplification mode design concept. The mechanical system model based on Simulink software showed that stroke of $203 \mathrm{~nm}$ was applied against $200 \mathrm{~N}$ mechanical friction. However, a backlash response was also observed due to the mass oscillation of all mechanisms of the proposed inchw orm motor. The effect of applying different mass amounts on the backlash response could be a design key solution for overcoming such backlash.

Acknowledgments: This project is funded by the Federal Ministry of Education and Research (BMBF) in Germany under the umbrella of Connected Health in Medical Mountains (CoHMed) of Furtwangen Unive rsity with grant number 13FH5I01IA.

Conflicts of Interest: The founding sponsors had no role in the design of the study; in the collection, analyses, or interpretation of data; in the writing of the manuscript, and in the decision to publish the results.

\section{References}

1. Huang, H.; Wang, Y. Wu. Design and Experimental Research of a Rotary Micro-Actuator Based on a She a ring Piezoelectric Stack. Micromachines 2019, 10, 96.

2. Zhao, B.; Fang, R.; Shi, W. Modeling of Motion Characteristics and Performance Analysis of an Ultra Precision Inchworm Motor. Materials 2020, 13, 3976.

3. Ma, L.; Jiang, C.; Xiao, J.; Wang, K. Design and Analysis of a Piezoelectric Inchworm Actuator. Journal of Micro-Robot 2014, 9:11-21.

4. Chen, X.; Li, M.; Zhang, H.; Lu, Q.; Lyu, S. Improvement on the Structure Design of a Kind of Linear Piezoelectric Motor with Flexible Drive-Foot. International Journal of Precision Engineering and Manufacturing 2020, 21:81-89.

(C) 2020 by the authors; licensee MDPI, Basel, Switzerland. This article is an open access article distribute $\mathrm{d}$ under the terms and conditions of the Creative Commons by Attribution (CC-BY) license (http://creativecommons.org/licenses/by/4.0/). 